\title{
hVH-5: A Protein Tyrosine Phosphatase Abundant in Brain that Inactivates Mitogen-Activated Protein Kinase
}

\author{
Karen J. Martell, *Audrey F. Seasholtz, Seung P. Kwak, Kristina K. Clemens, \\ and Jack E. Dixon \\ Department of Biological Chemistry, and ${ }^{*}$ Mental Health Research Institute, University of Michigan, \\ Ann Arbor, Michigan, U.S.A.
}

\begin{abstract}
A novel protein tyrosine phosphatase [homologue of vaccinia virus $\underline{H} 1$ phosphatase gene clone $\underline{5}$ (hVH-5)] was cloned; it shared sequence similarity with a subset of protein tyrosine phosphatases that regulate mitogen-activated protein kinase. The catalytic region of $\mathrm{hVH}-5$ was expressed as a fusion protein and was shown to hydrolyze $p$-nitrophenylphosphate and inactivate mitogen-activated protein kinase, thus proving that $\mathrm{hVH}-5$ possessed phosphatase activity. A unique proline-rich region distinguished hVH-5 from other closely related protein tyrosine phosphatases. Another feature that distinguished $\mathrm{hVH}-5$ from related phosphatases was that $\mathrm{hVH}-$ 5 was expressed predominantly in the adult brain, heart, and skeletal muscle. In addition, in situ hybridization histochemistry of mouse embryo revealed high levels of expression and a wide distribution in the central and peripheral nervous system. Some specific areas of abundant $\mathrm{hVH}-5$ expression included the olfactory bulb, retina, layers of the cerebral cortex, and cranial and spinal ganglia. $\mathrm{hVH}-5$ was induced in PC12 cells upon nerve growth factor and insulin treatment in a manner characteristic of an immediate-early gene, suggesting a possible role in the signal transduction cascade. Key Words: Phosphatase-Immediate-early gene-Mitogen-activated protein kinase.
\end{abstract}

J. Neurochem. 65, 1823-1833 (1995).

A key step in the regulation of several neuronal processes is the tyrosine phosphorylation of proteins (Hanks et al., 1988; Ullrich and Schlessinger, 1990; Aaronson, 1991). For instance, in the PC12 cell neuronal model system, nerve growth factor (NGF) has been shown to activate a series of kinases, culminating with differentiation of the cell into an electrically excitable sympathetic neuron-like phenotype (Greene and Tischler, 1976; Dichter and Tischler, 1977; Gomez and Cohen, 1991). Cellular studies using inhibitors indicated that protein tyrosine phosphatases (PTPases) also play a pivotal role in this NGF-mediated response in PC12 cells (Gomez et al., 1990; Wu and Bradshaw, 1993). Although one PTPase has been cloned from PC12 cells to date (Pan et al., 1993; Yan et al., 1993), no PTPases known to be involved in the NGF-induced differentiation pathway have been cloned. Indeed, only a few PTPases specific to neuronal tissues as a whole have been characterized. These include phosphatases that are present in the striatum (Lombroso et al., 1991), in the olfactory epithelium (Walton et al., 1993), and in axons of the embryonic central nervous system in Drosophila melanogaster (Yang et al., 1991). The relative position of these PTPases in signal transduction cascades and possible substrates for these PTPases remain unidentified.

Recently, a new subset of PTPases has been described. These PTPases dephosphorylated not only phosphotyrosine but also phosphoserine and phosphothreonine, thereby broadening the scope of potential substrates that are regulated by these PTPases (Guan et al., 1991; Sun et al., 1993; Ward et al., 1994). These PTPases are accordingly referred to as having dual specificity. Recent work has shown a role for these PTPases in the cell cycle and in the mitogenactivated protein kinase (MAPK) cascade (Millar and Russell, 1992; Zheng and Guan, 1993; Hannon et al., 1994; Ward et al., 1994). One member of this subclass, the cell division cycle protein $25(\operatorname{cdc} 25)$, has been

Resubmitted manuscript received March 6, 1995; accepted April $20,1995$.

Address correspondence and reprint requests to Dr. J. E. Dixon at the University of Michigan Department of Biological Chemistry, Medical Science I M5416, Ann Arbor, MI 48109, U.S.A.

Abbreviations used: cdc25, cell division cycle protein 25 ; cdc2, cell division cycle protein $2 ; \mathrm{CH} 2, \operatorname{cdc} 25$ homology domain $2 ; \operatorname{cdk} 5$, cyclin-dependent kinase 5 ; el 6.5 , embryonic day 16.5 ; hVH-1, -4 , or -5 , homologue of vaccinia virus $\underline{H} 1$ phosphatase gene clone 1,4 , or 5; GST, glutathione $S$-transferase; KAP, cyclin-dependent kinaseassociated phosphatase; MAPK, mitogen-activated protein kinase; NGF, nerve growth factor; PAC-1, phosphatase of activated cells; PKA, protein kinase A; PKC, protein kinase C; pNPP, $p$-nitrophenylphosphate; PTPase, protein tyrosine phosphatase; SH3, src homology domain 3; VH1, vaccinia virus phosphatase $\mathrm{Hl}$ gene; VHR, VH1-related phosphatase; Vn, sodium orthovanadate; YVH1, yeastrelated VH1.

The sequence reported in this paper has been deposited in the GenBank database (accession no. U27193). 
shown to initiate cell proliferation by dephosphorylating the premitosis promoting factor complex (Millar and Russell, 1992). Conversely, other phosphatases within this subclass, phosphatase of activated cells (PAC-1) (Ward et al., 1949) and homologue of vaccinia virus $\underline{\mathrm{H}} 1$ phosphatase gene clone 1 (hVH-1) (Sun et al., 1993; Zheng and Guan, 1993), appeared to inhibit cell proliferation by dephosphorylating and inactivating MAPK, the kinase that mediates many early cellular response cascades (Clarke, 1994; Marshall, 1994). Transfection of hVH-1 into NIH 3 T3 cells slowed cell division and altered cell morphology into an enlarged, multinucleated phenotype (Noguchi et al., 1993), further supporting the conjecture that this PTPase may function to inhibit cellular proliferation. PAC- 1 and $\mathrm{hVH}-1$ have both been shown to be immediate-early gene products induced by mitogenic stimuli, indicating that this effect on cell growth may be articulated early in the cell response (Charles et al., 1992; Rohan et al., 1993). hVH-1 was most abundant in lung, although it was also found at lower levels in many tissues including placenta, liver, pancreas, heart, skeletal muscle, and brain (Charles et al., 1992; Carrasco and Bravo, 1993; Kwak et al., 1994). It is interesting that PAC-1 was expressed predominantly in hematopoietic tissues, including spleen, thymus, bone marrow, and tumor cell lines of $\mathrm{B}$ and $\mathrm{T}$ cell origin (Rohan et al., 1993).

The relatively restricted distribution of the hematopoietic tissue-specific PTPase, PAC-1, suggested that a similar dual-specificity PTPase may be present in neural tissue. To identify such PTPases expressed predominantly in neuronal tissues, the genes for several novel dual-specificity PTPases were cloned (Martell et al., 1994) and portions of the genes were used to examine the tissue-specific expression of the corresponding RNA by northern blot analysis. This report describes the cloning and characterization of one of these PTPases, hVH-5, whose expression is abundant in brain. The deduced amino acid sequence of hVH-5 indicated that it was most similar to the dual-specificity PTPase subset. This subset was distinguished by regions of sequence similarity to cdc 25 within the amino terminus. The proline-rich carboxy terminus found in hVH-5 is unique within the dual-specificity subclass and this feature could be involved in the regulation of hVH-5 function, location, or stability. In situ hybridization histochemistry of mouse embryonic day 16.5 (e16.5) tissue demonstrated that hVH-5 mRNA was observed throughout the CNS with high levels in regions of the olfactory bulb, the retina, and several layers of the cerebral cortex. The pattern of $\mathrm{hVH}-5$ expression within the cerebral cortex suggested a trend such that hVH-5 expression was high in differentiating (or differentiated) neurons in developing cortex, but not detectable in rapidly proliferating cells. hVH-5 was also abundantly expressed in the PNS of the e16.5 mouse as indicated by intense hybridization to cranial and spinal ganglia. In addition, hVH-5 appeared to play a possible role in the neurotrophic or trophic factormediated response in PC12 cells, as demonstrated by the rapid induction of $\mathrm{hVH}-5 \mathrm{mRNA}$ expression in PC12 cells upon treatment with NGF or insulin, respectively.

\section{EXPERIMENTAL PROCEDURES}

\section{Materials}

Unless otherwise specified, all chemicals were supplied by Sigma Chemical Company. Radioisotopes were obtained from Amersham Corporation. Dulbecco's modified Eagle's medium, serum, and restriction endonucleases were purchased from Bethesda Research Laboratories. Enzymatic digestions with these restriction endonucleases were carried out according to the manufacturer's instructions. T7 and T3 RNA polymerases and the RNA Transcription kit were purchased from Boehringer-Mannheim Corporation and reactions were carried out according to the manufacturer's specifications.

\section{Isolation of cDNA clones}

Approximately one million independent recombinants of a human fetal brain cDNA library (Stratagene) were screened using a 1.3-kb fragment of genomic clone hVH-4 (Martell et al., 1994). This fragment was prepared by digesting hVH4 with Apal. The hVH-4 fragment was radiolabeled with a multiprime labeling system (Amersham Corporation) and $\left[\alpha{ }^{32} \mathrm{P}\right] \mathrm{dCTP}(3,000 \mathrm{Ci} / \mathrm{mmol})$ to a specific activity of 1.5 $\times 10^{9} \mathrm{cpm} / \mu \mathrm{g}$ of DNA. Procedures for screening the library were described previously (Martell et al., 1991). Twelve hybridization-positive clones were obtained in duplicate. Four of these cDNA clones were identical to the genomic sequence hVH-4. The remaining clones were overlapping fragments that all corresponded to a cDNA clone ( $\mathrm{hVH}-5)$ that had $93 \%$ nucleotide identity to hVH-4. The full-length hVH-5 clone was produced by subcloning the two longest overlapping clones into the EcoRI site of pBluescript II (Stratagene) to make the construct pBSctot. Sequencing was carried out using a Sequenase kit (United States Biochemical) or using the Ladderman kit (Takara) for G/C-rich regions.

A partial fragment of the mouse homologue of hVH-5 was cloned from an e16.5 mouse library (Stratagene). Approximately two million independent recombinants were screened as described above and 30 clones were obtained. Pst I digestion of one of the clones released a 500-bp fragment of the mouse homologue of hVH-5. Sequencing of this fragment revealed that it contained the catalytic domain of the phosphatase. This fragment was subcloned into the PstI site of pBluescript II (Stratagene) to create pMSE5P.

\section{Protein expression and activity analysis}

The enzymatic activity of the mouse homologue of hVH5 was assessed by expression of the cDNA as a glutathione $S$-transferase (GST) fusion protein. The entire 500-bp PstI fragment of mouse hVH-5 was chosen for the protein expression experiments because this fragment corresponded closely to the region of a related phosphatase, PAC-1, that was successfully expressed as a truncated phosphatase (Ward et al., 1994). The 500-bp Pst I fragment (from pMSE5P, described above) was subcloned in frame into pGEX-KG (Guan and Dixon, 1991) and production of the fusion protein was performed as described (Guan and Dixon, 1991). pNitrophenylphosphate (pNPP) and MAPK activity assays 
were carried out as described (Zheng and Guan, 1993) except that assay incubations were carried out for $90 \mathrm{~min}$. Activated MAPK was a generous gift of Dr. K. L. Guan, University of Michigan, Ann Arbor, MI, U.S.A. Inhibition studies included the PTPase inhibitor sodium orthovanadate ( Vn) at a final concentration of $2 \mathrm{mM}$, as described previously (Zheng and Guan, 1993 ).

\section{Human tissue northern blot analysis}

The human multitissue northern blot and the human brain region-specific northern blot were both purchased from Clontech. Each lane contained $1 \mu \mathrm{g}$ of poly(A) + mRNA of the various tissues or brain regions shown in each lane. The blots were prehybridized for $2 \mathrm{~h}$ in $5 \%$ sodium dodecyl sulfate, $320 \mathrm{mMNa} \mathrm{NPO}_{4}, 80 \mathrm{~m} M \mathrm{NaH}_{2} \mathrm{PO}_{4}, \mathrm{pH} 7.3,1 \mathrm{mM}$ EDTA, $1 \mathrm{mg} / \mathrm{ml}$ bovine serum albumin, and $50 \%$ formamide (Bethesda Research Laboratories). Blots were then hybridized overnight in the same solution at $70^{\circ} \mathrm{C}$ with an antisense riboprobe. The antisense riboprobe was prepared by first linearizing pBSctot template (construct described under Isolation of cDNA clones) with BamHI. The riboprobe was labeled to a specific activity of $1 \times 10^{6} \mathrm{cpm} / \mu \mathrm{g}$ with the linearized template, T3 RNA polymerase, $\left[\alpha-{ }^{32} \mathrm{P}\right]$ UTP $(3,000 \mathrm{Ci} / \mathrm{mmol})$, and the RNA Transcription kit. The blots were washed and exposed as described by Martell et al. (1991).

\section{In situ hybridization histochemistry}

Mouse embryos (e16.5) were frozen in 2-methylbutane and stored at $-80^{\circ} \mathrm{C}$. The embryos were sectioned $(15-\mu \mathrm{m}$ sections ) and processed for in situ hybridization histochemistry as described previously (Herman et al., 1989), except that deproteination was performed batchwise in $100 \mathrm{~m} M$ Tris- $\mathrm{HCl}, \mathrm{pH} 8.0,50 \mathrm{mM}$ containing $0.5 \mathrm{mg} / \mathrm{ml}$ proteinase $\mathrm{K}$ for $5 \mathrm{~min}$ at $37^{\circ} \mathrm{C}$. The plasmid pMSE5P, described under Isolation of cDNA clones, was linearized with BamHI for preparation of antisense cRNA. Alternatively, for preparation of sense cRNA, template pMSE5P was linearized with HindIII. Antisense and sense riboprobes were produced from the appropriate template with T3 or T7 RNA polymerase, respectively, using the RNA Transcription kit and ${ }^{35}$ S-UTP $(3,000 \mathrm{Ci} / \mathrm{mmol})$. Probe was diluted in hybridization buffer described previously (Herman et al., 1989) to yield $2,000,000 \mathrm{dpm} / 35 \mu \mathrm{l}$ of buffer and incubated at $54^{\circ} \mathrm{C}$ for 16-20 h. Sections were rinsed as described (Herman et al., $1989)$ prior to batchwise digestion with RNase A (200 $\mu \mathrm{g} /$ $\mathrm{ml}$ ) (Boehringer-Mannheim Corporation) in $10 \mathrm{~m} M$ Tris$\mathrm{HCl}$, pH 8.0 , and $0.5 \mathrm{M} \mathrm{NaCl}$ at $37^{\circ} \mathrm{C}$ for 60 min. Washing and dehydration were performed as described (Herman et al., 1989). Sections were exposed to Kodak XAR-5 x-ray film (International Biotechnologies Incorporated) for $24 \mathrm{~h}$ and then emulsion dipped in Kodak NTB2 nuclear emulsion (International Biotechnologies Incorporated) for 2-3 weeks of exposure.

\section{PC12 cell culture and northern blot analysis}

PC12 cells were grown in Dulbecco's modified Eagle's medium with $10 \%$ horse serum and $5 \%$ fetal calf serum in a humidified $8 \% \mathrm{CO}_{2}$ incubator. Cells were treated with NGF $(100 \mathrm{ng} / \mathrm{ml})$ or insulin $(100 \mathrm{n} M)$ (Bethesda Research Laboratories) for the time periods indicated. RNA extraction was carried out as described previously (Martell et al., 1992) and northern blotting was carried out using techniques described (Maniatis et al., 1982). Each lane contained approximately $8 \mu \mathrm{g}$ of poly (A) + mRNA. Hybridization conditions were the same as described above under Human tissue northern blot analysis. The riboprobe was labeled to a specific activity of $1 \times 10^{6} \mathrm{cpm} / \mu \mathrm{g}$ and was prepared as described for the in situ hybridization histochemistry experiments, except that $\left[\alpha-{ }^{32} \mathrm{P}\right]$ UTP $(3,000 \mathrm{Ci} / \mathrm{mmol})$ was used. The blots were washed and exposed as described (Martell et al., 1991).

\section{RESULTS}

\section{Molecular cloning and sequence analysis of hVH-5}

To search for a variety of tissue-specific, dual-specificity PTPases, a human genomic library was screened under various stringencies using a partial fragment of hVH-1 cDNA (Martell et al., 1994). A genomic library was chosen because many previously cloned dual-specificity PTPases are immediate-early gene products and therefore may be expressed only transiently and not present in a cDNA library. The genes for multiple novel dual-specificity PTPases were identified using this strategy and mapped to their respective chromosomes (Martell et al., 1994). The gene reported as hVH-4 was determined to be a pseudogene (authors' unpublished observations), but hybridization of a northern blot with a fragment of hVH-4 suggested the presence of a similar PTPase that was expressed abundantly in brain (data not shown).

To isolate the cDNA encoding this PTPase, a human fetal brain cDNA library was screened. The $2.5-\mathrm{kb}$ cDNA encoded a protein with an open reading frame of 625 amino acids (Fig. 1); the putative initiator, methionine, was identified by sequence comparison to the optimal sequence as defined by Kozak (1981, 1984 ). The presence of adenine or guanine at position -3 and a guanine at position +4 , the most invariant aspects of the Kozak consensus sequence, was observed in the context of the putative initiator methionine. The position of this methionine relative to the catalytic domain was similar to two other dual-specificity PTPases, PAC- 1 and hVH-1 (Keyse and Emslie, 1992; Rohan et al., 1993). The clone was named hVH5 to group it systematically with related phosphatases (Martell et al., 1994). Consensus sequences for mRNA destabilization, AUUUA (Shaw and Kamen, 1986), were observed in the 3' untranslated region of $\mathrm{hVH}$ 5 (Fig. 1). No polyadenylation sequence or poly (A) tail was observed in the human hVH-5 clone. However, a poly (A) tail was found in the mouse homologue of hVH-5, just 3 ' to the end of the human cDNA (data not shown). Examination of the deduced amino acid sequence of $\mathrm{hVH}-5$ revealed multiple putative recognition sequences for protein kinases. These included protein kinase A (PKA), protein kinase C (PKC), and the proline-directed family of kinases, specifically MAPK and the cell cycle regulatory kinase, cell division cycle protein $2(\operatorname{cdc} 2)$ (Fig. 1).

The encoded hVH-5 protein had the signature sequence (V/I)HCxAGxxR(S/T) (amino acids 244253 ) found in all protein tyrosine phosphatases (Fischer et al., 1991). Alignment of the deduced amino 
$\begin{array}{cccc}20 & 40 & 60 & 80\end{array}$ $\begin{array}{rrrr}140 & 160 & 180 & 200\end{array} 2$

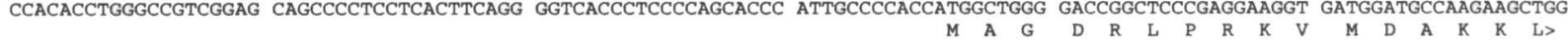
$\begin{array}{rrrr}260 & 280 & 300 & 320 \\ \text { CCAGCCTGCTGCGGGGCGG CCTGGGGGCCGCTGGTCAT CGACAGCCGCTCCTTCGTGG AGTACAACAGCTGGCATGTG CTCAGCTCCGTCAACATCTG CTGCTCCAAGCTGGTGAAGC }\end{array}$

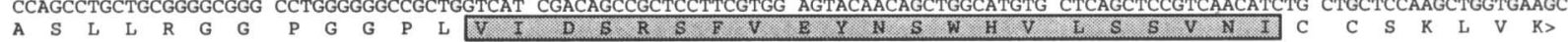
$\begin{array}{lllll}380 & 400 & 420 & 440 & 460\end{array}$

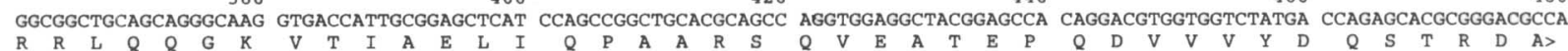
$\begin{array}{lllll}500 & 520 & 540 & 560 & 600\end{array}$

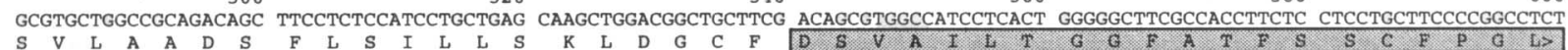

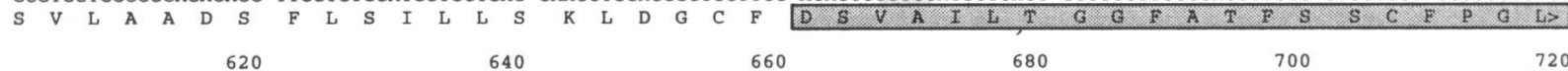
GCGAGGGCAAGCCTGCTGCC CTGCTACCCATGAGCCTCTC CCAGCCCTGCCTGCCTGTGC CCAGCGTGGGCTTGACCCGC ATCCTGCCTCACCTCTACCT GGGCTCGCAGAAGGACGTCC

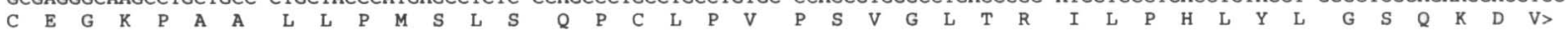
$\begin{array}{rrrr}740 & 760 & 780 & 800\end{array}$

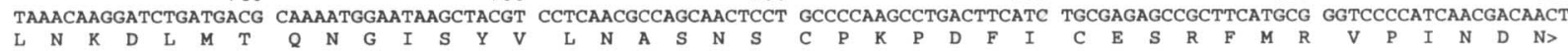
$\begin{array}{llll}860 & 880 & 900 & 920\end{array}$

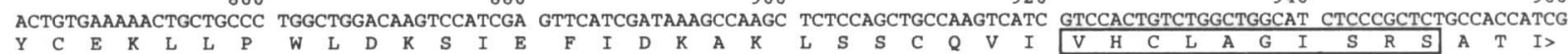
$\begin{array}{lllll}980 & 1000 & 1020 & 1040 & 1060\end{array}$ CCATCGCCTACATCATGAAG ACCATGGGCATGTCCTCCGA CGACGCCTACAGGTTCGTGA AGGACAGGCGCCCGTCCATC TCGCCCAACTTCAACTTCCT GGGCCAGCTGCTGGAGTACC

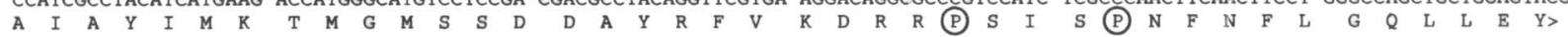
$\begin{array}{crrrrr}* & 1100 & 1120 & 1140 & 1160 & 1180\end{array}$

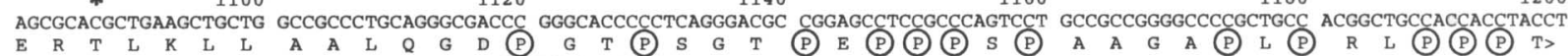
$\begin{array}{llllll}1220 & 1240 & 1260 & 1280 & 1300\end{array}$

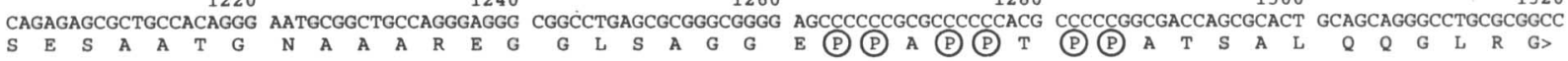

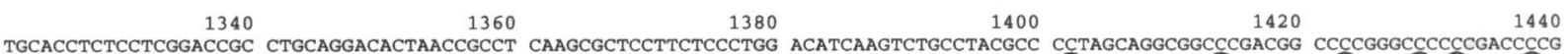

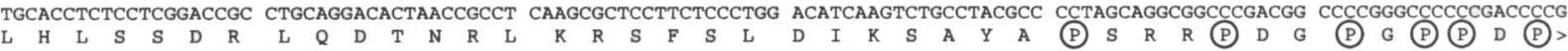
$\begin{array}{rrrrr}1460 & 1480 & 1500 & 1520 & 1540\end{array}$

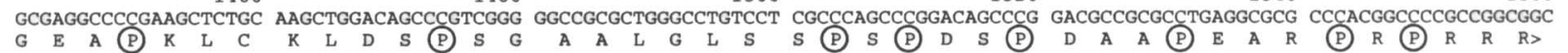

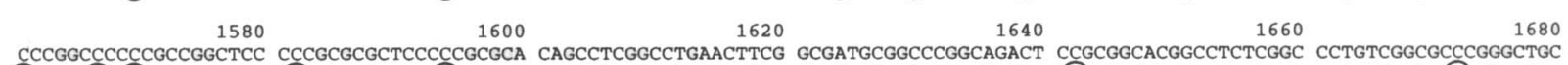

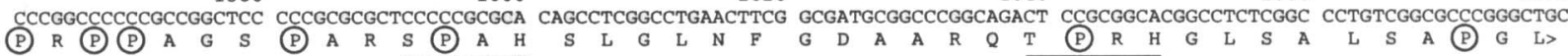
$\begin{array}{rrrrr}1700 & 1720 & 1740 & 1760 & 1780\end{array}$

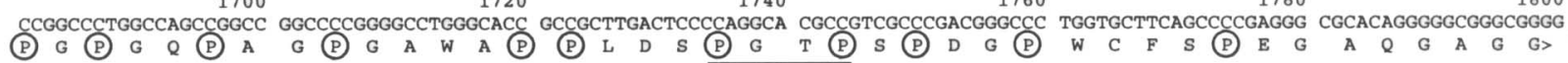

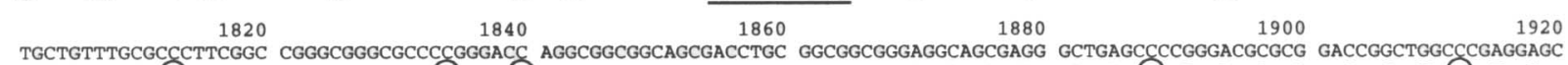

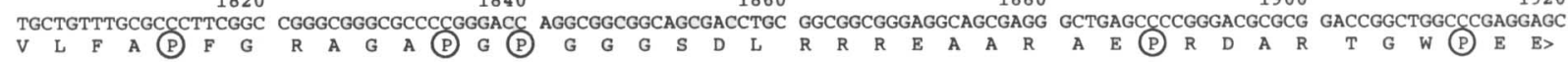
$\begin{array}{rrrr}1940 & 1960 & 1980 & 2000\end{array}$

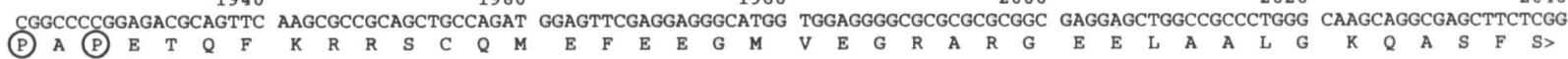
$\begin{array}{rrrr}2060 & 2080 & 2100 & 2120\end{array}$ GCAGCGTGGAGGTCATCGAG GTGTCCTGACCCCTCCGCTG CCCTCGGCCCCGCCGCCCGC AGCCAGGCCCGTTATAAATG TATATTATATATAATGCAAA GAAAGTAAATGGTTTTACT
$\mathrm{G}$ S V E V I E V S

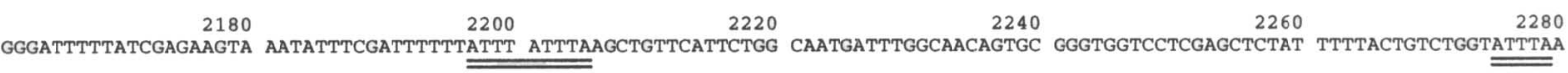

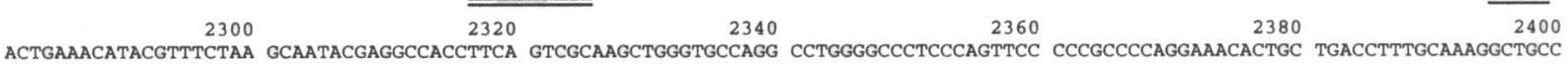
$\begin{array}{rrrr}2420 & 2440 & 2460 & 2480\end{array}$

FIG. 1. Nucleotide and deduced amino acid sequence of $\mathrm{hVH}-5$. Nucleotides are numbered above the sequence beginning at the first nucleotide of the isolated cDNA, and amino acids are numbered at the right beginning with the putative initiator Met. The amino acid residues that comprise the conserved catalytic PTPase domain are highlighted with an open box. The two domains that are conserved with cdc25 ( $\mathrm{CH} 2$ domains) are shaded. A putative phosphorylation site for PKA, RRPS (amino acids 279-282), is indicated by a solid black dot, and the recognition sequence for PKC phosphorylation, RTLK (amino acids 298-301), is indicated by an asterisk. Putative phosphorylation sites for proline-directed kinases are underlined. These included consensus phosphorylation sites for MAPK (ClarkLewis et al., 1991), such as PGTP (residues 310-313 and 315-318), whereas SPAH (467-480) and TPRH (483-486) conformed to the recognition site for phosphorylation by cdc2 (Moreno and Nurse, 1990). Consensus sequences for mRNA destabilization (ATTA) (Shaw and Kamen, 1986) are double underlined. All proline residues are encircled. 


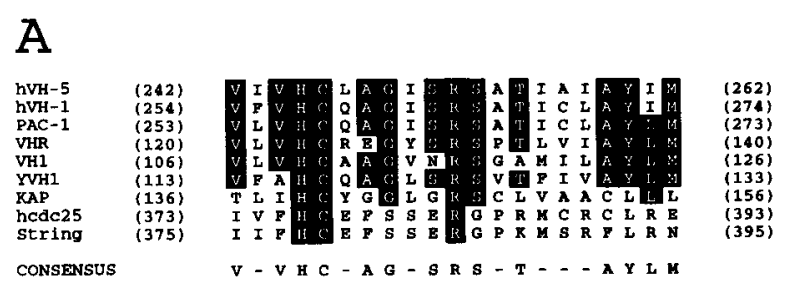

B

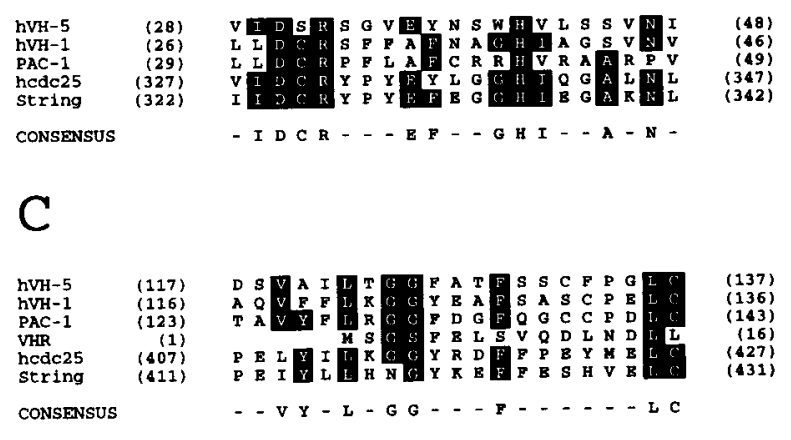

$\mathrm{D}$

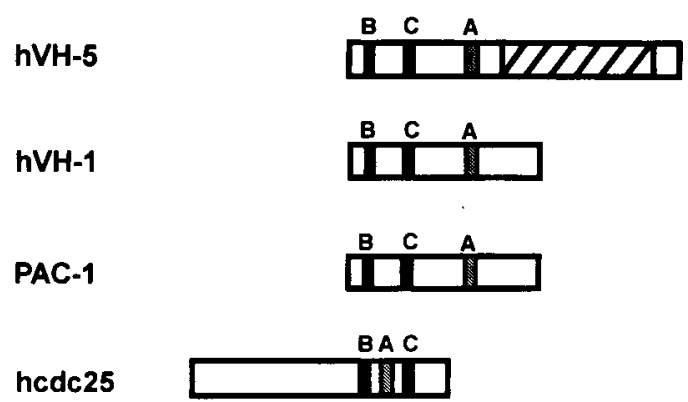

FIG. 2. Alignment and schematic representation of conserved domains of hVH-5 with dual specificity PTPases. Schematic is drawn to scale. Amino acid positions of the conserved domains are indicated in parentheses at the beginning and end of the sequence and a consensus line is given beneath each alignment. Conserved amino acid residues are highlighted. A: Alignment of the conserved active site sequence. $\mathbf{B}$ and $\mathbf{C}$ : Alignments of the first and second $\mathrm{CH} 2$ domains, respectively. D: Schematic representation of $\mathrm{hVH}-5, \mathrm{hVH}-1, \mathrm{PAC}-1$, and human cdc25, aligned with respect to the first $\mathrm{CH}_{2}$ domain $(B)$. The bands labeled $A, B$, and $C$ on the schematic correspond to the alignments above. The hatched box in $\mathrm{hVH}-5$ represents the prolinerich domain.

acid sequence of $\mathrm{hVH}-5$ with other protein sequences in the Genbank database demonstrated that $\mathrm{hVH}-5$ was most similar to dual-specificity PTPases. In Fig. 2A, the catalytic domain of hVH-5 was aligned with several closely related dual-specificity PTPases. Overall, hVH-5 shared the greatest amino acid identity with PAC-1 (36\% amino acid identity) (Rohan et al., 1993) and hVH-1 (34\% amino acid identity) (Keyse and Emslie, 1992). Other related PTPases included vaccinia virus phosphatase H1 gene (VH1) (Guan et al., 1991 ); yeast-related VH1 ( YVH1), a PTPase induced in yeast during nitrogen starvation (Guan et al., 1992);
VH1-related phosphatase (VHR), a human fibroblast PTPase (Ishibashi et al., 1992); and cyclin-dependent kinase-associated phosphatase (KAP), a PTPase that interacts with cyclin-dependent kinases (Hannon et al., 1994). Other related dual-specificity PTPases included the mitotic inducers, human cdc25 (Sadhu et al., 1990), and the Drosophila homologue of cdc25, string (Edgar and O'Farrell, 1989). Inspection of the residues surrounding the active site of the aligned PTPases revealed an area of extended homology $C$ terminal to the signature PTPase catalytic domain. This additional area of homology extended the signature sequence of the active site for a subset of dual-specificity PTPases to VxVHCxAGxSRSxTxxxAYLM (Fig. 2A).

Further examination of the amino acid sequence revealed two motifs conserved within a different subset of the dual-specificity PTPases. These motifs in hVH5 were underlined in Fig. 1 and aligned with similar regions of the homologous PTPases in Fig. 2B and C. These two regions have been referred to as cdc25 homology domains 2 ( $\mathrm{CH} 2$ domains) (Keyse and Ginsburg, 1993; Kwak et al., 1994). The location of the $\mathrm{CH} 2$ domains ( $\mathrm{B}$ and $\mathrm{C}$ ) relative to the catalytic domain (A) is shown schematically for hVH-5, PAC1, hVH-1, and cdc25 in Fig. 2D. While the $\mathrm{CH} 2$ domains of hVH-5, PAC-1, and hVH-1 were amino terminal to the catalytic domain, the $\mathrm{CH} 2$ domains of cdc25 surrounded the catalytic domain. These $\mathrm{CH} 2$ domains were also observed in other novel PTPases found in the genomic library screen (Martell et al., 1994).

One of the most distinctive features of the hVH-5 protein was the abundance of proline residues, predominantly in the carboxy terminus, accounting for $12 \%$ of the total amino acids. This proline-rich segment was not similar to any sequences in the Genbank database. Proline-rich segments have been examined recently as src homology domain 3 (SH3) binding sites (reviewed in Morton and Campbell, 1994); however, hVH-5 did not interact with any SH3 domains tested (data not shown).

Another possible role for the proline-rich domain may be protein stabilization. An abundance of proline (P), glutamate (E), serine ( $S$ ), and threonine (T) residues was commonly found in the most rapidly degraded eukaryotic proteins. Such a concentration collection of these residues (in no specific order) is referred to by the acronym PEST, although aspartic acid (D) is also manifold. The half-life of proteins containing PEST sequences is frequently $1 \mathrm{~h}$ or less (Rogers et al., 1986). The carboxy terminus of hVH-5 contains numerous proline $(17 \%)$, glutamate $(6 \%)$, serine $(9 \%)$, threonine $(3 \%)$, and aspartate $(4 \%)$ residues, which may serve as a PEST sequence to limit the half-life of the protein in the cell. The putative PEST regions, the amino acid locations of these regions, and the associated PEST score are shown in Table 1. The PEST scores were graded such that the higher the value, the greater the likelihood that the region was a 
TABLE 1. PEST sequences of $h V H-5$

\begin{tabular}{lll}
\hline $\begin{array}{c}\text { PEST } \\
\text { score }\end{array}$ & $\begin{array}{c}\text { Amino acid } \\
\text { residue \# }\end{array}$ & \multicolumn{1}{c}{ PEST sequence } \\
\hline 3.11 & $320-350$ & KLLAALQGDPGTPSGTPEPPPSPAAGAPLPR \\
7.54 & $443-469$ & KLDSPSGAALGLSSPPDSPDAAPEAR \\
5.27 & $589-603$ & RTGWPEEPAPETQFK \\
\hline
\end{tabular}

The PEST sequences and scores were determined according to a PESTFIND program kindly provided by Dr. M. Rechsteiner, University of Utah. The locations of the PEST sequences are indicated by the amino acid numbers corresponding to the sequence of hVH-5 shown in Fig. 1. Generally, the higher the PEST score, the greater the likelihood that the region is a PEST sequence. For comparison, the PEST scores of sequences within rapidly degraded proteins range from 0.1 for p53 to 24.4 for myc (Rogers et al., 1986).

PEST region. The values calculated for the PEST scores indicated that the proline-rich regions of $\mathrm{hVH}-$ 5 were likely to be PEST sequences. PEST sequences have been identified in only two other PTPases: PEP (Matthews et al., 1992) and PTP-PEST (Yang et al., 1993). Protein half-lives were not reported for PEP and PTP-PEST; however, the calculated PEST scores ranged from 4.43 to 13.34 for PEP and from 2.74 to 5.27 for PTP-PEST.

A portion of mouse $\mathrm{hVH}-5$ was cloned in addition to the human sequence. The mouse clone was $88 \%$ identical at the nucleotide level and $97 \%$ identical in amino acid sequence compared with the corresponding area of the human sequence ( amino acid residues 134306) (data not shown). The amino acid differences between the mouse and the human clone were threonine (mouse) for alanine (human) at position 143 on the human clone; proline for leucine at 145 ; serine for proline at 146; isoleucine for valine at 211; and serine for threonine at 299. These amino acid substitutions were not within the extended signature sequence of the active site, indicating that this particular area is highly conserved between species.

\section{Phosphatase activity determination}

Enzymatic activity was assessed by expressing a portion of hVH-5 as a GST fusion protein. Successful production of the fusion protein was achieved by expression of a truncated phosphatase corresponding to amino acid residues 134-306. This truncation eliminated the putative PEST sequence, as well as a portion of the amino terminus, which had interfered with expression in bacterial systems (authors' unpublished observations ). A similar truncation scheme was used successfully by other laboratories to express PAC-1 (Ward et al., 1994).

hVH-5 was able to hydrolyze the artificial substrate pNPP (Fig. 3A), which is used extensively as an indicator of phosphatase activity (Fischer et al., 1991). Vn, which has been shown to be an inhibitor of PTPases (Fischer et al., 1991), abolished the ability of hVH-5 to hydrolyze pNPP (Fig. 3A). GST protein alone was unable to hydrolyze pNPP over the time period of the assay (data not shown).

Phosphatases that are most similar to hVH-5 have been shown to inactivate MAPK by dephosphorylation on tyrosine and threonine residues (Zheng and Guan, 1993; Ward et al., 1994). MAPK activity was therefore assayed after incubation in the presence of increasing quantities of hVH-5. The addition of hVH-5 abolished MAPK activity in a concentration-dependent fashion (Fig. 3B). Addition of Vn inhibited the ability of hVH5 to inactivate MAPK (data not shown).

\section{Tissue and brain region distribution}

The specific expression pattern of $\mathrm{hVH}-5 \mathrm{mRNA}$ in human tissues was examined by northern analysis. hVH-5 mRNA was highly abundant in brain, although it was also present in heart and skeletal muscle (Fig. 4A). It is noteworthy that these tissues share the common feature of being electrically excitable and able to propagate an action potential. However, analysis of mouse tissue mRNA indicated that hVH-5 was also present in lung (data not shown).

Two transcript sizes of 5.5 and $2.5 \mathrm{~kb}$ were observed (Fig. 4A). The message of approximately $5.5 \mathrm{~kb}$ was more abundant than the message at $2.5 \mathrm{~kb}$, except in the case of skeletal muscle, where the two messages

\section{A}
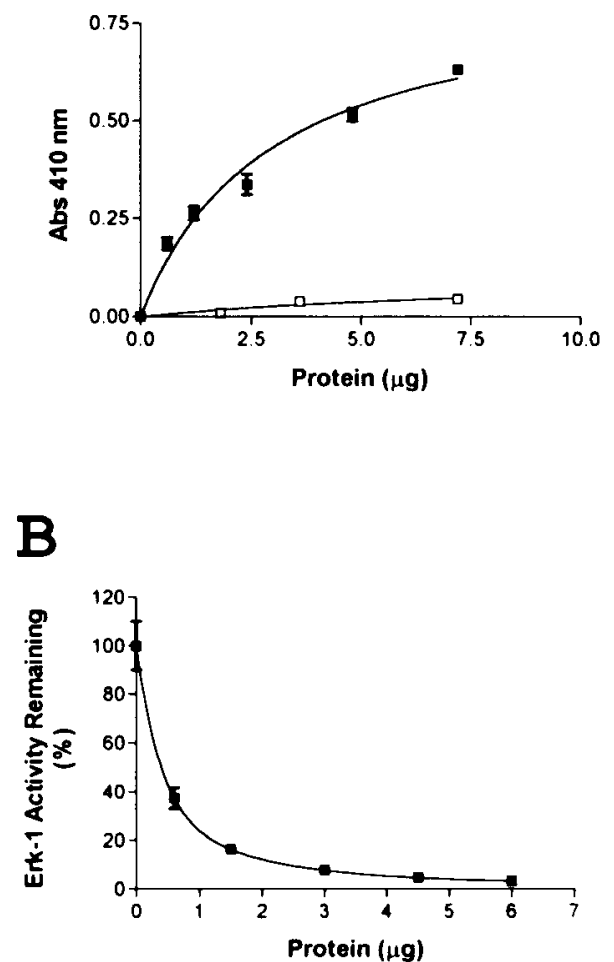

FIG. 3. Investigation of recombinant GST-hVH-5 enzymatic activity. A: Hydrolysis of pNPP in the presence ( $\square$ ) or absence (ם) of $2.5 \mathrm{mM} \mathrm{Vn}$. B: Inactivation of MAPK. Activated MAPK $(0.1$ $\mu \mathrm{g}$ ) was used in each point. Each point on the graph indicates a mean of triplicate experiments and error bars indicate the SEM. The amount of $\mathrm{hVH}-5$ added to each assay is indicated as micrograms of protein on the $x$-axis. 
A

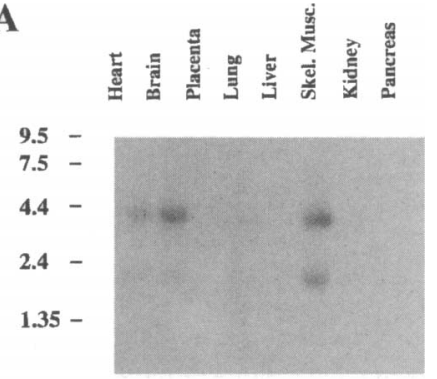

B
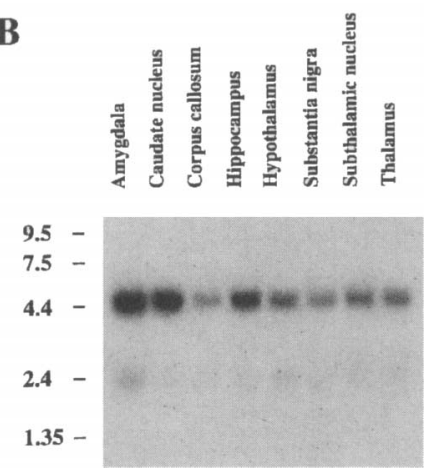

FIG. 4. Northern blots displaying tissue and brain region expression of hVH-5. A: Tissue distribution of hVH-5 mRNA. B: Brain region distribution of $\mathrm{hVH}-5$. The migration of RNA size markers is shown to the left.

appeared to be expressed equivalently. The size of the smaller message was consistent with the size of the cDNA clone reported in Fig. 1. The larger message has to date resisted further cloning attempts.

Because hVH-5 is very abundant in human brain, northern blot analysis was used to determine whether the message was confined to a particular brain region, or expressed ubiquitously in the CNS (Fig. 4B). hVH5 message was highly expressed in all human brain regions shown, including amygdala, caudate nucleus, corpus callosum, hippocampus, hypothalamus, substantia nigra, subthalamic nucleus, and thalamus. As was noted in heart and skeletal muscle (Fig. 4A), two mRNAs of 5.5 and $2.5 \mathrm{~kb}$ were observed, and again, the message of approximately $5.5 \mathrm{~kb}$ was more abundant than the message at $2.5 \mathrm{~kb}$.

\section{In situ hybridization analysis}

Northern analysis revealed the expression pattern of hVH-5 in adult tissues and brain-specific regions. However, the hVH-5 cDNA had been cloned from a fetal human brain library. To examine the expression of hVH- 5 at a single time in embryonic development, we examined the distribution pattern of hVH-5 mRNA with the mouse homologue of hVH-5 in sagittal and parasagittal sections from e16.5 mouse using in situ hybridization histochemistry. This time point was chosen because it was the same time in development as the cDNA library source used for cloning embryonic mouse hVH-5.
An autoradiograph of two adjacent sections hybridized to antisense (Fig. 5A) and sense (Fig. 5B) probes revealed that $\mathrm{hVH}-5$ was abundantly expressed in the developing neural system. In this sagittal section of e16.5 mouse, hVH-5 was readily observed in the developing brain, spinal cord, and cranial and spinal ganglia. No specific signal was detected in sections hybridized with sense cRNA probe, demonstrating the specificity of hybridization. Photomicrographs of emulsion-dipped sections further refined the examination of hVH-5 expression in the CNS and PNS (Figs. 6 and 7). hVH-5 mRNA was observed in almost all regions of the e16.5 brain, including developing cerebral cortex, forebrain, diencephalon (hypothalamus and thalamus), midbrain, pons, medulla, and cerebellar primordium (Fig. 6A). Expression levels appeared similar throughout most regions of the developing mouse brain, with the exception of very high levels in the olfactory bulb (Fig. 6B). Levels of hVH-5 expression also appeared to vary across the layers of developing cerebral cortex (Fig. $6 \mathrm{C})$. The rapidly proliferating neuroepithelial cells at the ventricular zone showed very little hVH-5 expres-

$\mathbf{A}$

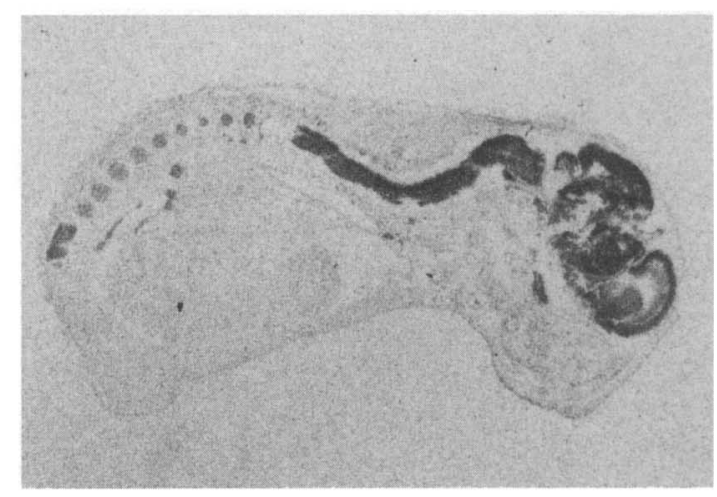

B

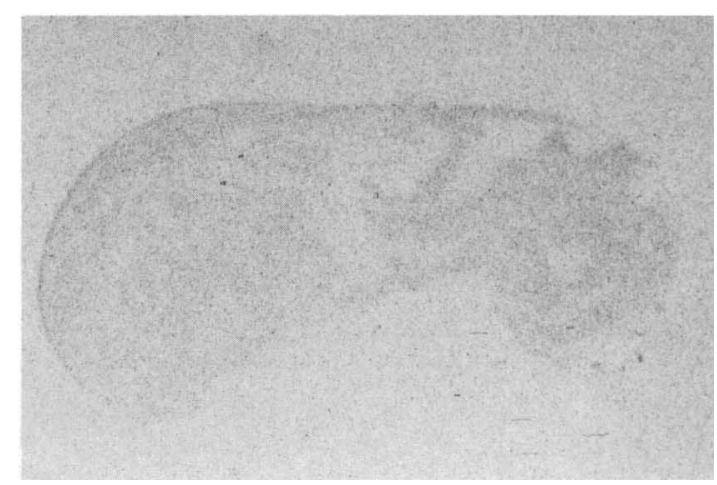

FIG. 5. In situ hybridization analysis of hVH-5 mRNA in mouse. Sagittal sections of mouse embryos (e16.5) were hybridized to antisense and sense ${ }^{35} \mathrm{~S}$-labeled $\mathrm{hVH}-5$ riboprobes. A: Autoradiogram of a sagittal section hybridized to antisense hVH-5 cRNA probe. B: Autoradiogram of the adjacent sagittal section hybridized to sense hVH-5 cRNA. 

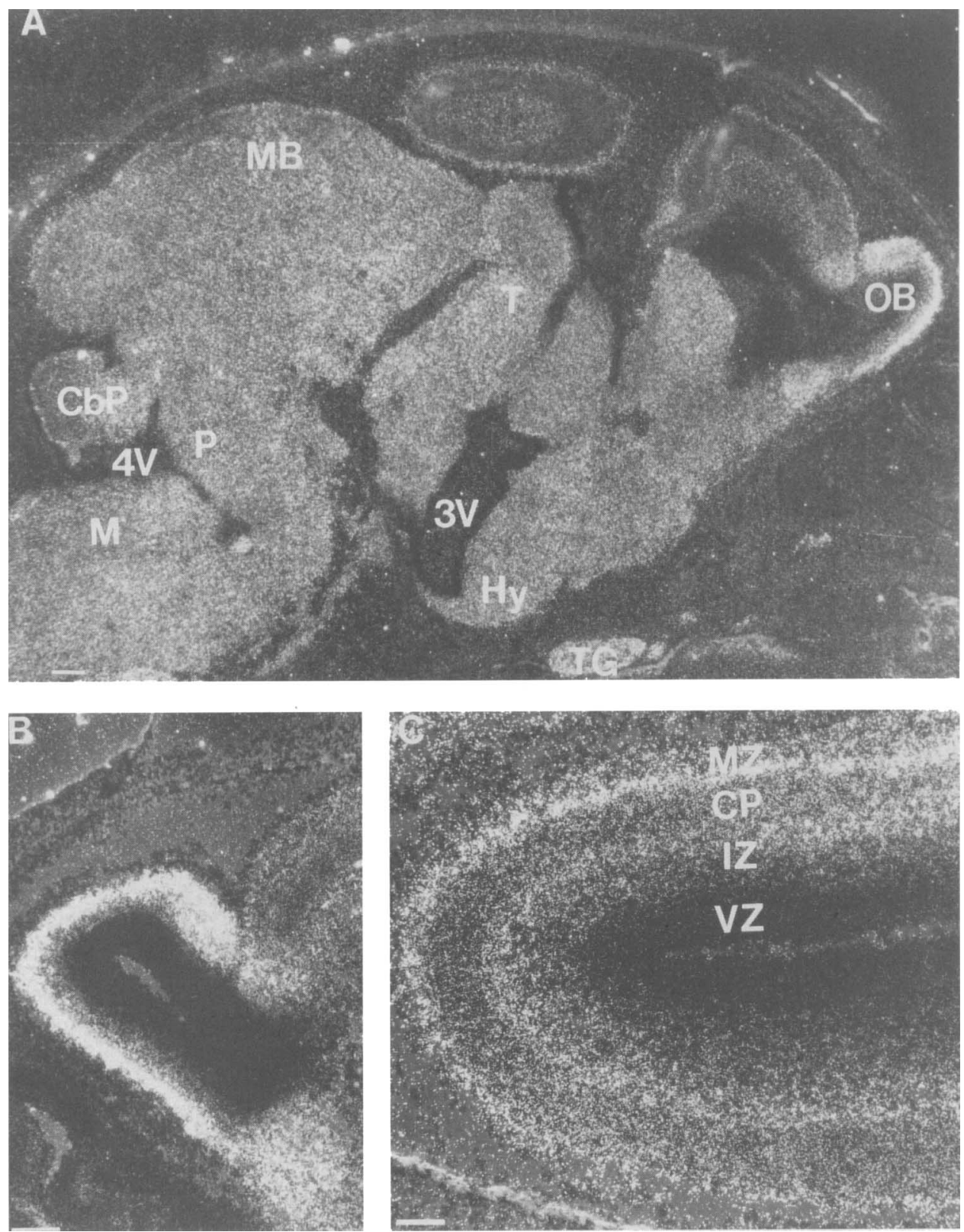

FIG. 6. Expression of hVH-5 mRNA in brain of e16.5 mouse embryo. Dark-field photomicrographs of sagittal sections hybridized with ${ }^{35}$ S-labeled antisense hVH-5 cRNA probe are shown. A: Developing mouse brain. B: Olfactory bulb. C: Developing cerebral cortex. Locations are as follows: OB, olfactory bulb; Hy, hypothalamus; T, thalamus; $3 \mathrm{~V}$, third ventricle; $\mathrm{MB}$, midbrain; $\mathrm{P}$, pons; $\mathrm{M}$, medulla; $\mathrm{CbP}$, cerebellar primordia; $4 \mathrm{~V}$, fourth ventricle; TG, trigeminal ganglion; VZ, ventricular zone; IZ, immediate zone; $\mathrm{CP}$, cortical plate; $\mathrm{MZ}$, marginal zone. Magnification bars represent $200 \mu \mathrm{m}(\mathrm{A}), 200 \mu \mathrm{m}(\mathrm{B})$, and $100 \mu \mathrm{m}(\mathrm{C})$.

sion, whereas the intermediate zone and marginal zone showed much higher levels of expression.

hVH-5 expression was also observed in the e 16.5 mouse retina and olfactory epithelium, both components of the CNS. The results suggested that high levels of hVH-5 mRNA were distributed in differentiating (or differentiated) ganglion cells of the retina, although this region could include developing amacrine, bipolar, or Mueller cells (Fig. 7A). Cells in the outer nuclear layer of the retina showed very little $\mathrm{hVH}-5$ expression.
Low levels of hVH-5 mRNA were also seen in the olfactory epithelium (data not shown). In addition, the spinal cord exhibited high levels of hVH-5 expression (Fig. 7B).

Within the e16.5 mouse PNS, hVH-5 mRNA was readily observed in the cranial ganglia and spinal ganglia. High levels of expression were observed in the trigeminal (Fig. 6A), facial, and glossopharyngeal ganglia. The dorsal root ganglia (Fig. 7C) also exhibited high levels of hVH-5 mRNA. 

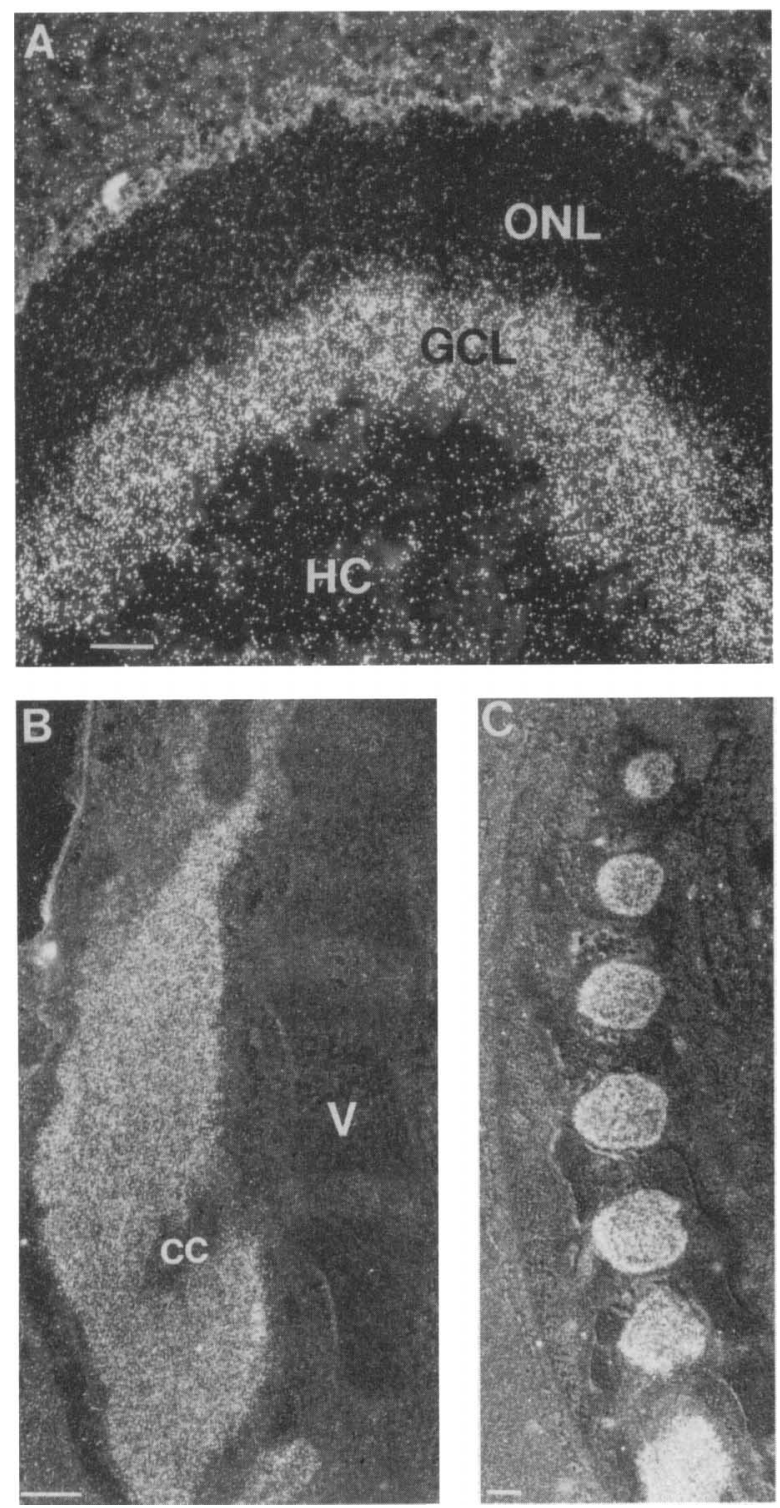

FIG. 7. Expression of $h \mathrm{VH}-5$ mRNA in developing CNS and PNS. Dark-field photomicrographs of sagittal sections hybridized with ${ }^{35}$ S-labeled antisense hVH-5 cRNA probe are shown. A: hVH-5 mRNA was highly expressed in differentiating (or differentiated) cells in the proximity of the developing ganglion cell layer. B: High levels of hVH-5 mRNA were observed in the spinal cord. C: The dorsal root ganglia exhibited high levels of hVH-5 mRNA. Locations are as follows: GCL, putative ganglion cell layer; $\mathrm{ONL}$, outer nuclear layer; $\mathrm{HC}$, hyaloid cavity; $\mathrm{V}$, cartilage primordium of vertebra; cc, central canal of spinal cord. Magnification bars correspond to $50 \mu \mathrm{m}(\mathrm{A}), 200 \mu \mathrm{m}(\mathrm{B})$, and $200 \mu \mathrm{m}(\mathrm{C})$.

\section{Expression in differentiating PC12 cells}

PAC-1 and hVH-1, the PTPases that were the most similar to hVH-5, have been shown to be immediateearly gene products that inactivate MAPK and may act as negative regulators of cell proliferation. It was therefore of interest to determine whether $\mathrm{hVH}-5$ was also an immediate-early gene product, thereby suggesting that $\mathrm{hVH}-5$ may perform a function similar to
PAC-1 and hVH-1. A number of cultured cell lines were examined for hVH-5 expression by northern blot analysis. Transcripts were observed in the human neuroblastoma cell lines SY5Y and Lan-5, and in rat pheochromocytoma PC12 cells (data not shown). PC12 cells were chosen as a model system because stimulation of these cells by the neurotrophin NGF or the trophic factor insulin triggers the MAPK pathway (Dikic et al., 1994). Accordingly, the early time course of $\mathrm{hVH}-5$ expression was examined in NGF- and insulin-treated PC12 cells.

hVH-5 mRNA levels increased within $30 \mathrm{~min}$ to a maximum induction in PC12 cells stimulated by NGF or insulin (Fig. 8). This elevation was sustained at the 1-h time point, and was reduced to pretreatment levels by $3 \mathrm{~h}$. mRNA levels increased again by $12 \mathrm{~h}$ posttreatment. The intensity of the signals on both blots was normalized to the abundance of cyclophilin, which was used as a control to account for variability in mRNA quantity between lanes and experiments (data not shown). Replicate experiments indicated a similar magnitude and time course of hVH-5 induction in PC12 cells treated with NGF or insulin (data not shown). These results mimicked the early time course of PAC-1 and hVH-1 induction (Charles et al., 1992; Rohan et al., 1993) and indicated that hVH-5 is an immediate-early gene product, which is also induced at later time points. This result was consistent with possible involvement of hVH-5 in inactivation of the MAPK cascade in PC12 cells.

\section{DISCUSSION}

$\mathrm{hVH}-5$ is a novel immediate-early gene product that was abundant in brain and had amino acid identity with the dual-specific subfamily of PTPases. Comparison of the primary sequence of $\mathrm{hVH}-5$ with related PTPases revealed an extended active site region: VxVHCxAGxSRSxTxxxAYLM that appeared to be a hallmark for a subset of dual-specificity PTPases (hVH-5, hVH1, PAC-1, VHR, VH1, YVH1). Additional areas of homology included the $\mathrm{CH} 2$ domains, which may play a role in defining substrate specificity or serve as a recruiting site for other proteins (Keyse and Ginsburg, 1993). The presence of the $\mathrm{CH} 2$ domains may also serve to define a restricted subset of the dual-specificity PTPases, because it was not present in other dualspecificity PTPases including VHR, VH1, YVH1, and KAP.

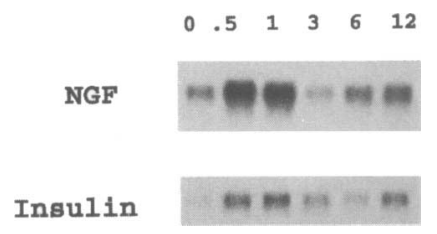

FIG. 8. Expression of $h \mathrm{VH}-5$ and mRNA in $\mathrm{PC} 12$ cells after incubation with NGF or insulin for $0,0.5,1,3,6$, or $12 \mathrm{~h}$. 
The presence of a proline-rich carboxyl extension in hVH-5 was unique among the dual-specificity PTPase subclass. The proline-rich tail may function as a regulatory domain for the abundance of $\mathrm{hVH}-5$ protein if the putative PEST regions within $\mathrm{hVH}-5$ are utilized as signals for rapid degradation within the cell. If this is the case, then the temporal abundance of hVH-5 may be regulated at multiple levels within the cell: hVH-5 may be controlled through PEST sequences at the protein level, and hVH-5 mRNA levels may be regulated through the presence of $\mathrm{mRNA}$ destabilization signals within the $3^{\prime}$ untranslated region (Fig. 1). The mRNA destabilization signal is commonly found in the mRNAs of transiently expressed genes and is proposed to be a signal sequence for mRNA degradation in certain immediate-early gene products (Shaw and Kamen, 1986). The identification of hVH-5 as an immediateearly gene product is consistent with the possibility that these sequences are indeed mRNA destabilization signals. These destabilization sequences were also found in the 3' untranslated regions of the related dualspecificity PTPases, PAC-1 and hVH-1 (Keyse and Emslie, 1992; Rohan et al., 1993). The presence of translational and transcriptional regulatory sequences suggests that hVH-5 mRNA expression may be tightly controlled and the encoded protein rapidly degraded. It is also noteworthy, as hVH-5 was so abundant in brain, that extended proline-rich regions have been observed in synaptic vesicle-associated proteins (Trimble and Scheller, 1988).

In the mouse embryo, hVH-5 was abundant throughout the CNS and PNS (Figs. 5-7). The ubiquitous and abundant nature of hVH-5 expression in the embryo brain was similar to the high levels of expression of hVH-5 mRNA observed throughout the adult human brain by northern blot analysis (Fig. 4B). Although hVH-5 appeared to be abundant in differentiating (or differentiated) neural cells in the embryo as well as in differentiated neural tissue in the adult, a complete developmental analysis will be necessary to draw conclusions about the nature of hVH-5 expression in neurogenesis. Such studies are currently underway in our laboratory with $\mathrm{hVH}-5$, and other investigators have already reported such developmental studies for externally regulated phosphatase, another dual-specificity PTPase (Carrasco and Bravo, 1993).

In situ hybridization histochemistry studies with the proline-directed cyclin-dependent kinase 5 (cdk5), a homologue of the cell cycle kinase cdc2, indicated that cdk5 was abundant in regions similar to hVH-5, including brain, spinal cord, and ganglia (Hellmich et al., 1992; Tsai et al., 1993). Several phosphorylation sites for proline-directed kinases were observed within the amino acid sequence of hVH-5 (Fig. 1) and, conceivably, the activity of $\mathrm{hVH}-5$ could be modified by any of these kinases. In addition, KAP, another dualspecificity PTPase with sequence similarity to hVH-5, interacts with cyclin-dependent kinases, including cdc2 (Hannon et al., 1994). Taken together, these data sug- gest that hVH-5 and cdk5 could interact with one another, and it would be of interest to explore a potential relationship between these two enzymes.

In PC12 cells, hVH-5 was induced by the neurotrophic factor NGF, and by the trophic factor insulin. Recent evidence suggests that in PC12 cells NGF and insulin mediate differentiation and proliferation, respectively, through common signaling mechanisms that involve the activation of MAPK. The differences in the cellular endpoints were thought to reside in differences in the magnitude and duration of MAPK activation and differences in the subcellular localization of MAPK (Dikic et al., 1994; Traverse et al., 1994). The factors that act to direct these differences in MAPK are poorly understood; however, it is possible that proteins such as $\mathrm{hVH}-5$ could influence the activation of MAPK or other key regulatory proteins, and thus play a pivotal role in the determination of proliferation or differentiation. The ability of hVH-5 to inactivate MAPK in vitro is consistent with such a role for $\mathrm{hVH}-5$. Extended examination of hVH-5 in PC12 cells may yield key observations about its function.

Acknowledgment: The authors wish to thank Drs. Carolyn Minth, Randy Stone, and Tim Angelotti for critical review of the manuscript. We thank Dr. Elizabeth Butch for providing activated MAPK. We greatly appreciate the PESTFIND program given to us by Dr. M. Rechsteiner, University of Utah. This work was supported by grants from the National Alliance for Research on Schizophrenia and Depression (NARSAD) (K.J.M.) and by National Institutes of Health grants DK18024 (J.E.D.) and DK-42730 (A.F.S.).

\section{REFERENCES}

Aaronson S. A. (1991) Growth factors and cancer. Science 254, $1146-1153$

Carrasco D. and Bravo R. (1993) Expression of the nontransmembrane tyrosine phosphatase gene erp during mouse organogenesis. Cell Growth Differ. 4, 849-859.

Charles C. H., Abler A. S., and Lau L. F. (1992) cDNA sequence of a growth factor-inducible immediate early gene and characterization of its encoded protein. Oncogene 7, 187-190.

Clarke P. (1994) Switching off MAP kinases. Curr. Biol. 4, 647650.

Clark-Lewis I., Sanghera J. S., and Pelech S. L. (1991) Definition of a consensus sequence for peptide substrate recognition by p44mpk, the meiosis-activated myelin basic protein kinase. $J$. Biol. Chem. 266, 15180-15184.

Dichter M. A. and Tischler A. S. (1977) Nerve growth factorinduced increase in electrical excitability and acetylcholine sensitivity of a rat pheochromocytoma cell line. Nature 268, 501504.

Dikic I., Schlessinger J,, and Lax I. (1994) PC12 cells overexpressing the insulin receptor undergo insulin-dependent neuronal differentiation. Curr. Opin. Biol. 4, 702-708.

Edgar B. A. and O'Farrell P. H. (1989) Genetic control of cell division patterns in the Drosophila embryo. Cell 57, 177-187.

Fischer E. H., Charbonneau H., and Tonks N. K. (1991) Protein tyrosine phosphatases: a diverse family of intracellular and transmembrane enzymes. Science 253, 401-406.

Gomez N. and Cohen P. (1991) Dissection of the protein kinase cascade by which nerve growth factor activates MAP kinases. Nature 353, 170-173.

Gomez N., Tonks N. K., Morrison C., Harmar T., and Cohen P. 
(1990) Evidence for communication between nerve growth factor and protein tyrosine phosphorylation. FEBS Lett. 271, 119 122.

Greene L. A. and Tischler A. S. (1976) Establishment of a noradrenergic clonal line of rat adrenal pheochromocytoma cells which respond to nerve growth factor. Proc. Natl. Acad. Sci. USA 73, 2424-2428.

Guan K. and Dixon J. E. (1991) Eukaryotic proteins expressed in Escherichia coli: an improved thrombin cleavage and purification procedure of fusion proteins with glutathione S-transferase. Anal. Biochem. 192, 262-267.

Guan K., Broyles S. S., and Dixon J. E. ( 1991) A Tyr/Ser protein phosphatase encoded by vaccinia virus. Nature 350, 359-362.

Guan K., Hakes D. J., Wang Y., Park H. D., Cooper T. G., and Dixon J. E. (1992) A yeast protein phosphatase related to the vaccinia virus VH1 phosphatase is induced by nitrogen starvation. Proc. Natl. Acad. Sci. USA 89, 12175-12179.

Hanks S. K., Quinn A. M., and Hunter T. (1988) The protein kinase family: conserved features and deduced phylogeny of the catalytic domains. Science 241, 42-52.

Hannon G. J., Casso D., and Beach D. (1994) KAP: a dual specificity phosphatase that interacts with cyclin-dependent kinases. Proc. Natl. Acad. Sci. USA 91, 1731-1735.

Hellmich M. R., Pant H. C., Wada E., and Battey J. F. (1992) Neuronal cdc2-like kinase: a cdc2-related protein kinase with predominantly neuronal expression. Proc. Natl. Acad. Sci. USA 89, 10867-10871.

Herman J. P., Schafer M. K. H., Young E. A., Thompson R., Douglass J., Akil H., and Watson S. J. (1989) Evidence for hippocampal regulation of neuroendocrine neurons of the hypothalamo-pituitary-adrenocortical axis. J. Neurosci. 9, 30723082

Ishibashi T., Bottaro D. P., Chan A., Miki T., and Aaronson S. A. (1992) Expression cloning of a human dual-specificity phosphatase. Proc. Natl. Acad. Sci. USA 89, 12170-12174.

Keyse S. M. and Emslie E. A. (1992) Oxidative stress and heat shock induce a human gene encoding a protein-tyrosine phosphatase. Nature 359, 644-647.

Keyse S. M. and Ginsburg M. ( 1993 ) Amino acid sequence similarity between CL100, a dual-specificity MAP kinase phosphatase, and cdc25. Trends Biochem. Sci. 18, 377-378.

Kozak M. (1981) Possible role of flanking nucleotides in recognition of the AUG initiator codon by eukaryotic ribosomes. Nucleic Acids Res. 9, 5233-5262.

Kozak M. (1984) Compilation and analysis of sequences upstream from the translational start site in eukaryotic mRNAs. Nucleic Acids Res. 12, 857-872.

Kwak S. P., Hakes D. J., Martell K. J., and Dixon J. E. (1994) Isolation and characterization of a human dual specificity protein-tyrosine phosphatase gene. J. Biol. Chem. 269, 3596-3604.

Lombroso P. J., Murdoch G., and Lerner M. (1991) Molecular characterization of a protein-tyrosine-phosphatase enriched in striatum. Proc. Natl. Acad. Sci. USA 88, 7242-7246.

Maniatis T., Fritsch E. F., and Sambrook J. ( 1982) Molecular Cloning: A Laboratory Manual, 1 st edit. Cold Spring Harbor Laboratories, Cold Spring Harbor, New York.

Marshall C. J. (1994) MAP kinase kinase kinase, MAP kinase kinase, and MAP kinase. Curr. Opin. Genet. Dev. 4, 82-89.

Martell K. J., Vatsis K. P., and Weber W. W. (1991) Molecular genetic basis of rapid and slow acetylation in mice. Mol. Pharmacol. 40, 218-227

Martell K. J., Levy G. N., and Weber W. W. ( 1992) Cloned mouse $N$-acetyltransferases: enzymatic properties of expressed Nat-1 and Nat-2 gene products. Mol. Pharmacol. 42, 265-272.

Martell K. J., Kwak S., Hakes D. J., Dixon J. E., and Trent J. M. (1994) Chromosomal localization of four human VH1like protein tyrosine phosphatases. Genomics 22, 462-464.

Matthews R. J., Bowne D. B., Flores E., and Thomas M. L. (1992) Characterization of hematopoietic intracellular protein tyrosine phosphatases: description of a phosphatase containing an $\mathrm{SH} 2$ domain and another enriched in proline-rich, glutamic acid-rich, serine-rich, and threonine-rich sequences. Mol. Cell. Biol. 12, $2396-2405$.

Millar J. B. A. and Russell P. (1992) The cdc25 M-phase inducer: an unconventional protein phosphatase. Cell 68, 407-410.

Moreno S. and Nurse P. (1990) Substrates for p34cdc2: in vivo veritas? Cell 61, 549-551.

Morton C. J. and Campbell I. D. (1994) Molecular 'Velcro'. Curr. Biol. 4, 615-617.

Noguchi T., Metz R., Chen L., Mattei M.-G., Carrasco D., and Bravo R. (1993) Structure, mapping, and expression of erp, a growth factor-inducible gene encoding a nontransmembrane protein tyrosine phosphatase, and effect of ERP on cell growth. Mol. Cell. Biol. 13, 5195-5205.

Pan M.-G., Rim C., Lu K. P., Florio T., and Stork P. J. S. (1993) Cloning and expression of two structurally distinct receptorlinked protein tyrosine phosphatases generated by RNA processing from a single gene. J. Biol. Chem. 268, 19284-19291.

Rogers S., Wells R., and Rechsteiner M. (1986) Amino acid sequences common to rapidly degraded proteins: the PEST hypothesis. Science 234, 364-368.

Rohan P. J., Davis P., Moskaluk C. A., Kearns M., Krutzsch H., Siebenlist U., and Kelly K. (1993) PAC-1: a mitogen-induced nuclear protein tyrosine phosphatase. Science 259, 1763-1766.

Sadhu K., Reed S. I., Richardson H., and Russell P. (1990) Human homolog of fission yeast cdc 25 mitotic inducer is predominantly expressed in G2. Proc. Natl. Acad. Sci. USA 87, 5139-5143.

Shaw G. and Kamen R. (1986) A conserved AU sequence from the $3^{\prime}$ untranslated region of GM-CSF mRNA mediates selective mRNA degradation. Cell 46, 659-667.

Sun H., Charles C. H., Lau L. F., and Tonks N. K. (1993) MKP-1 (3CH134), an immediate early gene product, is a dual specificity phosphatase that dephosphorylates MAP kinase in vivo. Cell $\mathbf{7 5}, 487-493$.

Traverse S., Seedorf K., Paterson H., Marshall C. J., Cohen P., and Ullrich A. (1994) EGF triggers neuronal differentiation of PC12 cells that overexpress the EGF receptor. Curr. Biol. 4, 694-701.

Trimble W. S. and Scheller R. S. (1988) Molecular biology of synaptic vesicle-associated proteins. Trends Neurosci. 11, $241-$ 242.

Tsai L.-H., Takahashi T., Caviness V. S., and Harlow E. (1993) Activity and expression pattern of cyclin-dependent kinase 5 in the embryonic mouse nervous system. Development 119, 10291040 .

Ullrich A. and Schlessinger J. (1990) Signal transduction by receptors with tyrosine kinase activity. Cell 61, 203-212.

Walton K. M., Martell K. J., Kwak S. P., Dixon J. E., and Largent B. L. (1993) A novel receptor-type protein tyrosine phosphatase is expressed during neurogenesis in the olfactory neuroepithelium. Neuron 11, 387-400.

Ward Y., Gupta S., Jensen P., Wartmann M., Davis R. J., and Kelly K. (1994) Control of map kinase activation by the mitogeninduced threonine/tyrosine phosphatase PAC-1. Nature 367, $651-654$.

Wu Y. Y. and Bradshaw R. A. (1993) Effect of nerve growth factor and fibroblast growth factor on PC12 cells: inhibition by orthovanadate. J. Cell Biol. 121, 409-422.

Yan H., Grossman A., Wang H., Deustachio P., Mossie K., Musacchio J. M., Silvennoinen O., and Schlessinger J. (1993) A novel receptor tyrosine phosphatase-sigma that is highly expressed in the nervous system. J. Biol. Chem. 268, 24880-24886.

Yang Q., Co D., Sommercorn J., and Tonks N. K. (1993) Cloning and expression of PTP-PEST: a novel, human, nontransmembrane protein tyrosine phosphatase. J. Biol. Chem. 268, 66226628 .

Yang X., Seow K. T., Bahri S. M., Oon S. H., and Chia W. (1991) Two Drosophila receptor-like tyrosine phosphatase genes are expressed in a subset of developing axons and pioneer neurons in the embryonic CNS. Cell 67, 661-673.

Zheng C. F. and Guan K. L. (1993) Dephosphorylation and inactivation of the mitogen-activated protein kinase by a mitogen-induced Thr/Tyr protein phosphatase. J. Biol. Chem. 268, 1611616119. 